Review

\title{
Exosomal tetraspanins mediate cancer metastasis by altering host microenvironment
}

\author{
Jun Lu ${ }^{3}$, Jun Li ${ }^{1,2}$, Shuo Liu ${ }^{1,2}$, Teng Wang ${ }^{1,2}$, Alessandro Ianni ${ }^{4}$, Eva Bober ${ }^{4}$, Thomas \\ Braun ${ }^{4}$, Rong Xiang ${ }^{1,2}$ and Shijing Yue ${ }^{1,2}$ \\ ${ }^{1}$ School of Medicine, Nankai University, Tianjin, China \\ ${ }^{2}$ The State International Science \& Technology Cooperation Base of Tumor Immunology and Biological Vaccines, Nankai \\ University, Tianjin, China \\ ${ }^{3}$ Department of General Surgery, Hefei Second People's Hospital, Hefei, China \\ ${ }^{4}$ Department of Cardiac Development and Remodeling, Max-Planck-Institute for Heart and Lung Research, Bad Nauheim, Germany \\ Correspondence to: Shijing Yue, email: shijingyve@nankai.edu.cn \\ Rong Xiang, email: rxiang@nankai.edu.cn \\ Keywords: tumor, cancer metastasis, tetraspanin, exosomes, cancer microenvironment
}

Received: August 04, $2016 \quad$ Accepted: April 05, $2017 \quad$ Published: July 10, 2017

Copyright: Lu et al. This is an open-access article distributed under the terms of the Creative Commons Attribution License 3.0 (CC BY 3.0), which permits unrestricted use, distribution, and reproduction in any medium, provided the original author and source are credited.

\section{ABSTRACT}

The metastases of malignant tumors develop through a cascade of events. The establishment of a pre-metastatic micro-environment is initiated by communication between tumors and host. Exosomes come into focus as the most potent intercellular communicators playing a pivotal role in this process. Cancer cells release exosomes into the extracellular environment prior to metastasis. Tetraspanin is a type of 4 times transmembrane proteins. It may be involved in cell motility, adhesion, morphogenesis, as well as cell and vesicular membrane fusion. The exosomal tetraspanin network is a molecular scaffold connecting various proteins for signaling transduction. The complex of tetraspanin-integrin determines the recruiting cancer exosomes to pre-metastatic sites. Tetraspanin is a key element for the target cell selection of exosomes uptake that may lead to the reprogramming of target cells. Reprogrammed target cells assist pre-metastatic niche formation. Previous reviews have described the biogenesis, secretion and intercellular interaction of exosomes in various tumors. However, there is a lack of reviews on the topic of exosomal tetraspanin in the context of cancer. In this review, we will describe the main characteristics of exosomal tetraspanin in cancer cells. We will also discuss how the cancer exosomal tetraspanin alters extracellular environment and regulates cancer metastasis.

\section{INTRODUCTION}

In tumor tissues, diverse types of non-cancer cells intermingle with heterogeneous cancer cells [1]. Nonmalignant cells and extracellular matrix (ECM) of the tumor are defined as the tumor stroma. Tumor progress is dependent on interactions within this complex micro-environment [2-4]. During the initiation, tumor growth beyond the threshold size of a few $\mathrm{mm}^{3}$ is largely determined by the regulation of tumor cell, non-tumor cell, angiogenesis, immunological system $[5,6]$. Cancer micro-environment is a complex system of many elements including the extracellular matrix, growth factors, angi-ogenic factors, stromal cells and inflammatory cells. Initiating the formation of new blood vessels is important for tumor growth and survival $[7,8]$. Bone-marrowderived cancer promoting cells (including haematopoietic progenitor cells and macrophages) express efficiently tumor growth factors. Bone-marrow-derived cancer promoting cells house tumor-specific pre-metastatic sites to prepare cancer pre-metastatic niche formation and metastasis [9-11].

Exosomes are derived from multi-vesicular bodies (MVBs) and contain mRNA, miRNA, as well as enriched tetraspanins and tetraspanin-associated proteins $[12,13]$. Tetraspanins are a major component of exosomes. It is 
likely that tetraspanins impact the functional activity of exosomes [14]. From current knowledge, there is strong evidence that tumor-derived exosomes play a major role in the interaction between tumor and host. The biogenesis and main physical characteristics have been defined with special focus on their oncogenic role [15-19]. The most important part of exosome biogenesis is the formation of intra-lumenal vesicles (ILVs) of MVBs. The endosomal sorting complex for transport (ESCRT-0, -I, II, -III) is a key component for exosome biogenesis. Numerous accessory proteins (including syndecan heparan) and syntenin, their cytoplasmic adaptor, control the formation of exosomes. ALIX, the interactive partner of syntenin, is required for exosome biogenesis [20-22].

The uptake of exosomes is mediated by the interaction between exosomal ligands and receptors of recipient cells. It is likely that there are several different mechanisms for the uptake of exosomes by target cells. Exosomes deliver content via internalization or by fusing the plasma membrane of the recipient cells [23-27]. Uptake of exosomes by target cells may accompany the transferring of mRNAs, miRNAs and proteins. The molecular activities dependent on exosomes uptake lead to reprogramming of the recipient cells [18, 28-30]. The published data has demonstrated that exosomal tetraspanins are involved in the mechanism of target cells uptake [31]. Besides carrying information into target cells, exosomes are also involved in remodelling the tumor micro-environment. Tumor exosomes confer new activities to stromal cells, endothelial cells, epithelial cells, fibroblasts and macrophages [32-34]. Exosomes-educated non-cancer cells promote tumor vascular permeability, immune escaping, angiogenic switch and highly drug resistant properties [35-41]. As signaling scaffolds, exosomal tetraspanins can induce a series of activations of target cells. Extracellular matrix is an important section of tumor micro-environment. Tumor exosomes remodeling extracellular matrix can prepare a path for cancer metastasis. Recently the conception, biogenesis, composition, secretion and cancer progress regulation of exosomes have been reviewed extensively [20-22, $35,42]$. The present review highlights tetraspanins that are involved in assembly of exosomes, exosomes uptake and exosomal tetraspanins regulating the alteration of cancer micro-environment.

\section{EXOSOMES: NEW INTERCELLULAR COMMUNICATORS}

\section{Discovery and original biogenesis of exosomes}

Exosomes was first discovered in vitro culture of sheep reticulocytes by Johnstone et al. in 1987 [43]. A decade later, a few investigations demonstrated that exosomes may be signaling carriers with proteins and bioactive molecules [44]. Additional studies showed further evidence that exosomes played the role of a communicator between cells [44-46]. Extracellular vesicles represent the large family of non-classical secretory vesicles, with microvesicles and exosomes being two sub-sets of this family.Exosomes - small extracellular vesicles of 30-100nm [42] - are secreted by multiple cells and distributed in all body fluids including blood, milk and urine. Exosomes are derived from the fusion of intraluminal vesicles of MVBs with the plasma membrane $[47,48]$. The molecular composition of exosomes indicates their origin from intraluminal vesicles $[49,50]$. Besides a set of common membrane and cytosolic molecules, the components of exosomes contain tetraspanins including CD9, CD37, CD53, CD63, CD81, CD82, CD151, and Tspan8 (CO-029/D6.1A). Exosomes harbour sub-sets of proteins contain integrins, ICAMs, MHC; vesicle transport associated molecules; cytoskeletal proteins; heat shock proteins (HSP); enzymes; signaling molecules, and so on $[42,51]$. ESCRT complexes (ESCRT-0, -I, II, -III), MFGE8, TSG101, flotillin, PDCD6IP (ALIX), and tetraspanins molecules (CD9, CD63, CD81) have been used as biomarkers of exosomes [52, 53].

A notable feature of exosomal proteins is the maintenance of functional activities including antigen presentation, peptide, and protein cleavage [54]. Valadi et al. indicated that exosomes contain mRNAs and miRNAs, that transfer to recipient cells with the corresponding function for intercellular regulation [55]. Exosomal mRNAs can be translated and miRNAs can mediate RNA-silencing in target cells. Gene transfer and gene silence mediated by exosomes are specific to target cells that are found in one specific but not in another type of cell [55-57]. In addition, the relative abundance of proteins, mRNAs and miRNAs differs between exosomes and donor cells [58]. This implies active sorting into MVBs. For proteins, this can be achieved by monoubiquitination, localized in cholesterol-rich membrane micro-domains, or oligomerization of a higher order $[47,59]$. Thus, exosomes constitute a most potent mode of intercellular communication that has become important for immunity, cell to cell spread of infectious agents and tumor progression $[49,54,60,61]$.

\section{Cargo and functional activities}

Exosomes from different sources exhibit distinct variation in their cargo of proteins and nucleic acids. Malignant cancer cells releasing the exosomes contain tumor- specific proteins. For instance, exosomes from ovarian cancer patients' ascites contain Her2/Neu and from melanoma secretion contain Mart1 [62]. Studies have shown that exosomes from the serum of ovarian cancer patients comprise 8 types of microRNAs (miR-21, miR-141, mir-200a, mir-200b, 200C, miR-203, mir-205, and miR-214). The level of microRNAs is similar in exosomes and the parent carcinoma cell, while it cannot 
be detected in health exosomes or cells. This investigation also indicates that exosomal miRNAs have diagnostic value for ovarian cancer [63]. In addition the diagnostic value of microRNAs has been found in other cancers [64] such as prostate cancer [65], esophageal squamous cell carcinoma [66] and malignant tumors in lungs [67].

The proteomic analysis demonstrates that package proteins of exosomes are specific and are completely different from cell apoptosis by the release of apoptotic bodies [68, 69]. The High Throughput Sequencing technology verifies that the miRNA is also subject to certain mechanisms rather than a random package of exosomes [70]. Many reports show exosomes involved in the regulation of a variety of physiological activities and pathological processes such as the immune system, tissue repair, nervous system of traffic [71], cardiovascular diseases, neuro-degenerative diseases as well as tumors [72]. These investigations have demonstrated that exosomes are active rather than passively produced by parent cells. They are the new messengers of intercellular communication.

\section{EXOSOMAL TETRASPANIN NETWORK}

\section{Structure of tetraspanin}

The transmembrane 4 super-family (TM4SF) or tetraspanins are small transmembrane proteins expressed in many species [73]. Tetraspanins are implicated in a diverse range of biologicalprocessesincludingphysiological cell adhesion, motility, activation and proliferation as well as pathological cancer metastasis and viral infection [74, 75]. The structure is postulated to cross the membrane 4 times during which there is a short $\mathrm{N}$ and $\mathrm{C}$ terminal tail; a small extracellular loop (ECL1) between transmembrane regions TM1 and TM2; a small intracellular loop (ICL) between TM2 and TM3; and a large extracellular loop (ECL2) between TM3 and TM4. The constant region of the ECL2 is responsible for dimerization of themselves, the variable region for associating non-tetraspanin molecular partners.

Key features of tetraspanins include a highly conserved ' $\mathrm{CCG}$ ' motif and an additional two or more cysteine residues in the ECL2 domain. TM regions contain polar residues to stabilize the tertiary structure [76]. Most tetraspanins are glycosylated in the region of ECL2, thought to impact tetraspanin function. Some tetraspanins may have palmitoylation cysteines in their transmembrane region. Palmitoylation is required for initiating tetraspanin-tetraspanin web formation [77]. Some tetraspanins contain a tyrosine-based sorting motif for intracellular compartment targeting that may lead to internalization via associated proteins [78] (Figure 1).

\section{Large protein complex of tetraspanins}

Tetraspanins associate with themselves as well as with numerous transmembrane and/or cytosolic proteins to assemble into complexes [12]. Tetraspanin enriched membrane micro-domains (TEMs) comprising tetraspanin complexes act as platforms for signalling transduction $[12,79,80]$. The strength of molecular interaction in tetraspanin complexes can be grouped into three types: type I is direct protein-protein interaction with stronger association; type II is tetraspanin dimerization and tetraspanin or integrin interaction with mild association; type III is tetraspanin and several kinases interactions with weaker association and stabilizing by palmitoylation $[76,79]$. Tetraspanins are associated with growth factor receptors, G-protein-coupled receptors (GPCRs), two members of immunoglobulin (Ig) super-family (EWI-F and EWI-2), and signal transduction molecules (PKC) $[81,82]$. Integrins are the most prominent molecular partners of tetraspanins including $\alpha 3 \beta 1, \alpha 4 \beta 1$, and $\alpha 6 \beta 1$. Tetraspanins act as molecular facilitators to modulate the functions of associated molecules [83]. They influence cell invasiveness by modulating MMP production and cell adhesion through regulating biogenesis and traffic of corresponding molecules $[84,85]$. Furthermore, several tetraspanins are also involved in the process of virus and parasite infections [86].

Exosomal tetraspanins engage in internalization, vesicle traffic and deviation from degradation in the proteasome $[62,87]$. Clathrin and intersectin2 are required for Tspan8 TEM internalization [87]. The function of tetraspanins in cargo loading of exosomes has not been fully investigated. The recruitment of miRNA in Tspan $8 \mathrm{kd}$ and CD151kd pancreatic cancer exosomes leads to minor differences. To address the question, the uniform exosomes need to be evaluated and scrutinized but not focusing on the single cell-type released exosomes. A few studies have concentrated on defining sub-populations of exosomes that facilitate exact targets [88].

In a cancer context, many studies indicate that Tspan8 promote cancer metastasis and angiogenesis [75, 89]. Furthermore, Tspan 8 mediates tumor cell motility and invasion $[72,90]$. Tetraspanin CD151 has been shown to be involved in tumor cell motility, invasion, metastasis and angiogenesis [69, 70]. CD9 is considered to be a tumor suppressor and facilitates tumor angiogenesis [76, 91]. Tetraspanin CD82 has been reported to be a tumor suppressor and a modulator of membrane heterogeneity $[77,92]$. Some investigations support that CD63 is a cancer metastasis suppressor, while the functional role of CD63 needs more evaluation [79, 93] (Table 1).

\section{EXOSOME ASSEMBLY AND TETRASPANINS INTERNALIZATION}

\section{Exosome assembly}

In most mammalian cells, portions of the plasma membrane are regularly internalized into a membrane bound compartment as endosomes [94]. Parts of the 
Table 1: The functional roles of tetraspanin in cancer

\begin{tabular}{|c|c|c|c|c|}
\hline Tetraspanin & Functional roles in cancer & $\begin{array}{l}\text { Detect in } \\
\text { exosomes }\end{array}$ & Key associated proteins & References \\
\hline CD151 & $\begin{array}{l}\text { Tumour cell motility, invasion, } \\
\text { metastasis, tumor initiation, promotion, } \\
\text { progression, and angiogenesis }\end{array}$ & yes & Integrins $\alpha 6 \beta 4, \alpha 6 \beta 1, \alpha 3 \beta 1$ & {$[35,71,72,127]$} \\
\hline Tspan8 & $\begin{array}{l}\text { Tumor growth, and angiogenesis, } \\
\text { tumour cell motility, invasion, } \\
\text { metastasis }\end{array}$ & yes & $\begin{array}{l}\text { E-cadherin, claudin } 7, \\
\text { EPCAM, } \alpha 6 \beta 4 \text { integrin, } \\
\text { CD44v6 and EWIF }\end{array}$ & $\begin{array}{l}{[35,74,77,91} \\
\quad 92,127]\end{array}$ \\
\hline CD9 & $\begin{array}{l}\text { Tumour metastasis suppressor, tumour } \\
\text { angiogenesis, lymphangiogenesis and } \\
\text { tumour growth }\end{array}$ & yes & $\begin{array}{l}\text { EWI2, EWIF EPCAM, HB- } \\
\text { EGF }\end{array}$ & {$[78,89,93]$} \\
\hline CD82 & $\begin{array}{l}\text { Suppressor of tumour migration, } \\
\text { invasion and metastasis }\end{array}$ & yes & Integrins $\alpha 6 \beta 4, \alpha 4 \beta 1, \alpha 3 \beta 1$ & {$[79,94]$} \\
\hline CD63 & Tumour metastasis suppressor & yes & $\begin{array}{l}\text { Integrins } \alpha 6 \beta 1, \text { TIMP1, } \\
\text { CD } 82, \text { CD } 9\end{array}$ & {$[81,95]$} \\
\hline
\end{tabular}

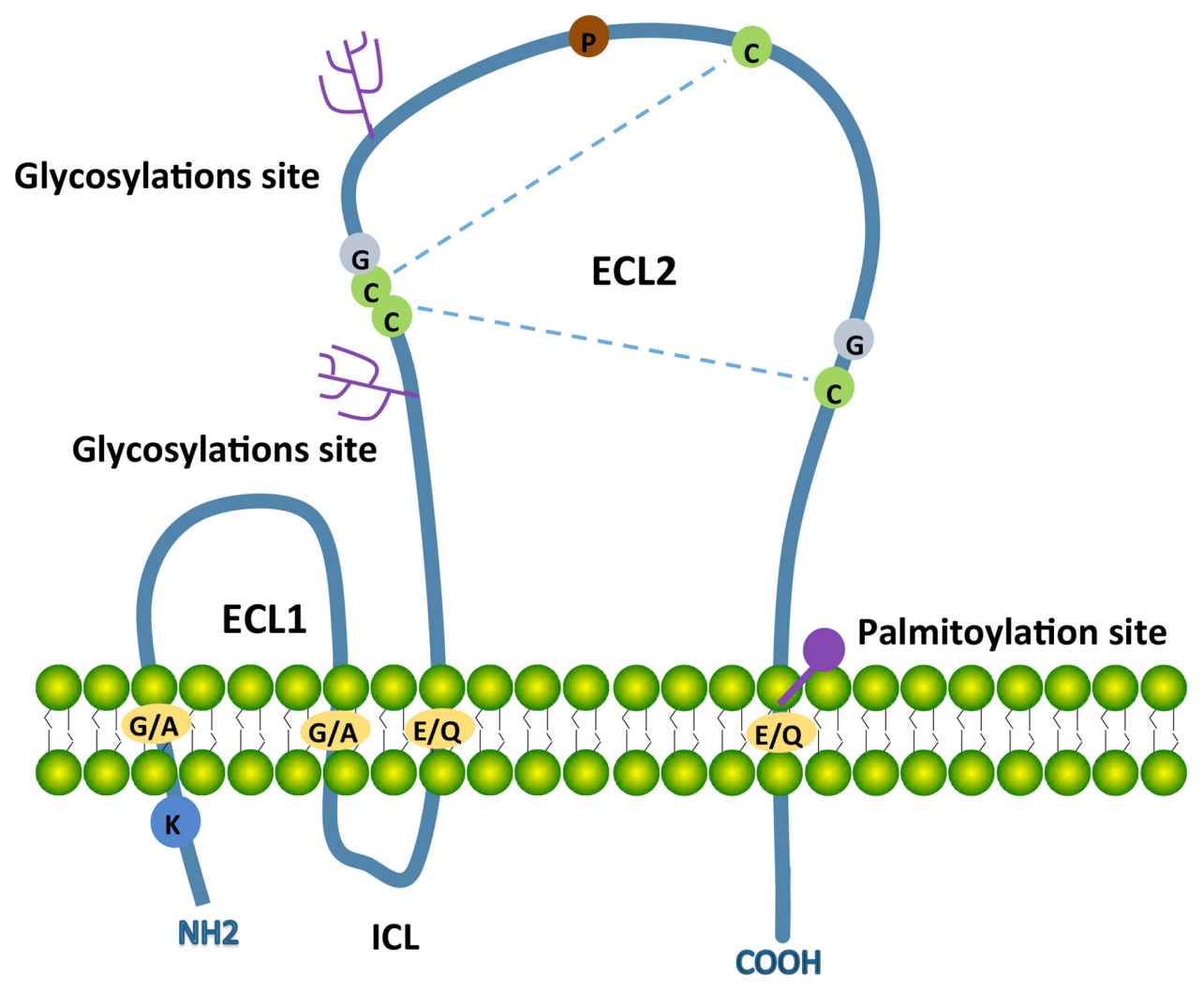

Figure 1: Schematic structure of most tetraspanins with key characteristics. Tetraspanins contain four transmembrane domains (TM1 to TM4), a small extracellular loop (ECL1), a small intracellular loop (ICL) and a large extracellular loop (ECL2). The N-terminal and C-terminal tails are intracellular. Transmembrane domains contain conserved polar/charged residues (orange circles), ECL2 contains a highly conserved CCG motif and additional two conserved cysteine residues (green circles). Two disulphide bridges (blue dotted lines) can form between these cysteine residues for the folding of ECL2. ECL2 contains two glycosylations site (pink trees) andTM4 has a putative palmitoylation site (pink circle). The conserved residues including one proline (brown circle) and two glycine (grey circles) locate in ECL2 and one lycine locates in N-terminal tail. 
membranes of some endosomes are subsequently internalized as smaller vesicles to become multi-vesicular bodies. The endosomes can become lysosomes or recycled back to the plasma membrane. Molecules from the plasma membrane can also become lysosomes for degradation or can be recycled back to the plasma membrane. Molecules are tagged with ubiquitin that can be recognized and sorted into lumenal vesicles by the ESCRTs [95-97]. Marked molecules in the lumen of endosomes will be degraded in lysosomes.

The multi-vesicular body fuses with the cell membrane and releases the intralumenal endosomal vesicles into extracellular space that become exosomes [98]. Multivesicular bodies, internalized with endosomes membrane, comprise the lipid rafts micro-domain with GPI-anchored proteins [99]. Endocytosis, mediated by clathrin-coated pits, regulates the internalization of many molecules as transferin and transferin receptors as lumenal vesicles $[100,101]$. Tetraspanins and tetraspanin-associated molecules locatedbyTEMs are sorted into small vesicles during the generation of MVBs. Cytoplasm protein can be internalized into MVBs via ESCRTs or ceramidedependent pathways [54] (Figure 2).

\section{Internalization of tetraspanins into exosomes}

Enrichment of tetraspanins in exosomes receives progressive attention in unraveling protein-protein and protein-lipid interactions, membrane dynamics and intracellular vesicle sorting $[74,78]$. Besides the clustering
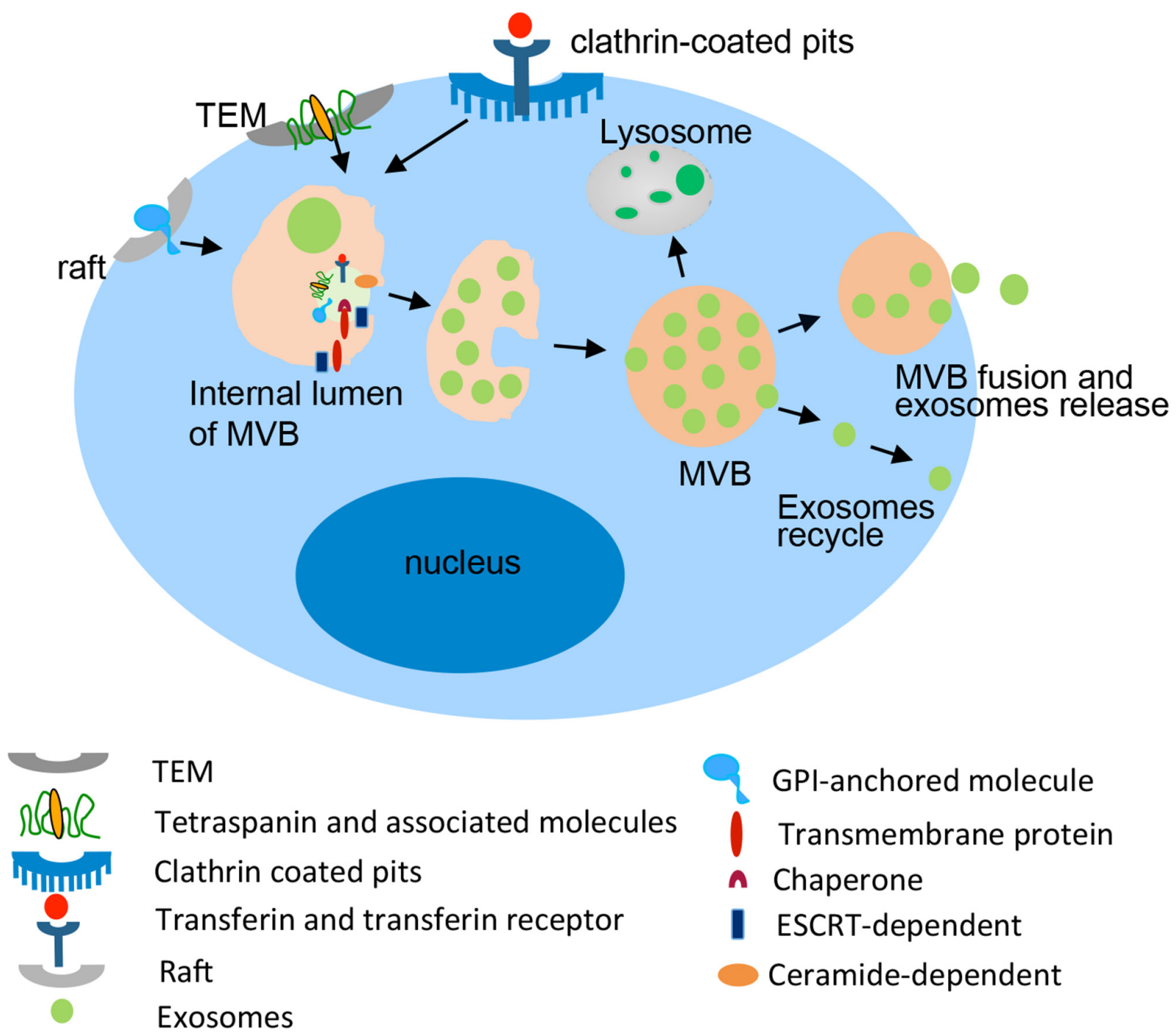

TEM

Tetraspanin and associated molecules

Clathrin coated pits

Transferin and transferin receptor

Raft

Exosomes

Figure 2: Biogenesis and composition of exosomes. Exosomes derived from the intralumenal vesicles (ILVs) of MVBs, which formed by the late endosomes with the membrane proteins sorted by TEM, Lipid rafts, Clathrin coated pits and the endocytosis of cytoplasmic molecules dependent on ESCRT or ceramide. The membrane of MVBs fuses with the plasma membrane resulting in the release of exosomes into the extracellular environment. 
potential ligands for signal transduction, tetraspanins may contribute to the assembly of exosomes. Tetraspanin internalization could influence the selectivity of exosomes assembly. For example, the content of proteins and mRNA is different in exosomes derived from the same cell line with or without transfection of Tspan8 cDNA [102]. Tetraspanins internalize into exosomes through specific molecules or motifs rather than through clathrin-coated pits and caveolae. A tyrosine-dependent internalization motif $(\mathrm{Yxx} \Phi)$ or the c-terminal tail of tetraspanins and associated molecules are suggested to facilitate TEMlocated tetraspanins internalization [78, 101, 103].

CD151 contains a sorting motif that allows rapid internalization. CD9 has no sorting motif whereas Tspan8 has one, but it is too closely located to bind the AP complex. In the resting cells, CD9 and Tspan8 are associated with CD151. The internalization of Tspan 8 and CD9 is accompanied by CD151 during PMA stimulation [87]. Intersection-2 (INS2), a multi-modular protein, gets involved in clathrin-mediated endocytosis with Tspan8-associating $\alpha 4$ or $\alpha 6 \beta 4$ complex [104]. It is known that Intersections (INSs) contain Eps15 domains that interact with dynamin and synaptojanin to promote endocytosis [105]. Tspan8 associating with INS2, dynamin, $\alpha 4$, or $\beta 4$ makes a major contribution to the generation of MVBs through the synthetic route and exosomes biogenesis [87, 102, 106].

\section{REGULATION OF TETRASPANINS IN EXOSOMES UPTAKE AND TARGET CELL REPROGRAMMING}

\section{Exosomal tetraspanin-complexes contribute to target selection}

Studies have shown that binding/uptake of exosomes in target cells is directed by specific mechanisms. It is likely that exosome uptake takes place in internalization-prone membrane micro-domains. New evidence indicates that the exosomal tetraspanin web is involved in the binding of target cell ligands and receptors. We have tested the uptake of 4 types of exosomes derived from pancreatic adenocarcinoma lines BSp73AS (AS) of a rat expressing different tetraspanins by target cells in vitro and in vivo (exosomes from AS, AS-Tspan8, AS-Tspan8/CD9n, AS-Tspan8/Integrinß4) [40]. The results demonstrated that the minor differences in the composition of exosomes cause the distinct uptake by target cells. AS-Tspan8 exosomes preferentially bind to rat endothelial cell, while the plus $\beta 4$ bind to stromal cell and AS exosomes bind to fibroblasts in vitro. The in vivo uptake of the selectivity of target cell is more surprisingly significant with differences between the 4 exosomes types [40, 107]. Pull-down experiments unraveled the membrane molecules involved in exosomes binding as MFGE8, CD44, CD49e, CD54, CD56, and CD106. In addition, phosphatidylserine (PS) was exposed on the outer membrane of exosomes support uptake [107]. Similar fusion/fission machineries are common for internalization of exosomes in parent cells and uptake of exosomes by recipient cells [47]. The lipid ligands and proteins of exosomes bind with cell surface receptors by activating the signal to deliver the factors to target cells [108].

Exosomes also can be involved in immunity regulation by the presentation of antigens or the transfer of antigens and major histocompatibility complex (MHC) molecules [52, 109]. The entrance of exosomes is dependent on transmembrane proteins including the interaction of ligands and receptors, the membrane fusion and the endocytosis process [110]. The subsequent mechanism of exosomes uptake is still poorly understood. Our studies demonstrate that the tetraspanin-integrin complex contributes to the selectivity of exosomes binding to target cells [40, 102]. David Lyden et al. revealed the exosomal integrins that are involved in target cell selection. Exosomal integrins activate the signalling pathway of Src phosphorylation and pro-inflammatory S100 gene expression. Integrins $\alpha 6 \beta 4$ and $\alpha 6 \beta 1$ are responsible for lung metastasis while integrin $\alpha v \beta 5$ is linked to liver metastasis (Figure 3) [111].

\section{Exosomes uptake promote target cell reprogramming}

Exosomes containing selected patterns of mRNA and miRNA imply that a dynamic regulation of gene expression can occur in the recipient cell. Besides the mRNA, a subset of miRNA can be transferred by exosomes derived from mouse embryonic stem cells to mouse embryonic fibroblasts in vitro [112]. Investigations suggested that transferring of miRNA mediated by exosomes may alter the expression of gene products in target cells. Cancer-associated fibroblasts (CAFs) exosomal miRNAs (miRNAs -21, -378e, and -143) can be delivered to breast cancer cells increasing the capacity of mammospheres formation, the expression of biomarker of stem cell and epithelial-mesenchymal transition (EMT) and ability of anchorage-independent cell growth [113]. The transfer of genetic information by exosomes play a pivotal role for reprogramming of target cells.

Aside from transfer miRNA, the exosomal content can be delivered into the target cell leading cell reprogramming. Zhou et.al demonstrated that exosomes derived from human embryonic stem cells can reprogram in malignant cancer cells. The cargo of exosomes are able to be transfered into target cancer cells and induce the expression of SOX2, OCT4 and Nanog. They also indicate that human embryonic stem cellderived exosomes played a dose-dependent decrease of cancer cell growth and tumorigenicity [114]. In tumor biology, exosomes can be regarded as signalosomes by stimulating tumor growth, angiogenesis, matrix remodeling, immune escape and metastasis. Exosomes can induce the expansion of regulatory $\mathrm{T}$ cells or apoptosis of $\mathrm{CD}^{+} \mathrm{T}$ cells to initiate tumor cell immune escape $[115,116]$. 


\section{CANCER MICRO-ENVIRONMENT ALTERATION MEDIATING METASTASIS}

\section{Exosomes mediate communication of stromal and cancer cells}

Cancer micro-environment is composed various cells including cancer associated fibroblast (CAFs), endothelial cells, tumor associated macrophages (TAMs), as well as extracellular matrix and other factor. Cancer metastasis is mediated by interactions among cancer cells and cancer micro-environment. Exosomes can regulate distant cellular communication, which modulate the crosstalking between the resident cell via various oncogenic signaling pathways.

Cancer cells derived exosomes transfer TGF- $\beta$ into CAFs to develop myofibroblastic phenotype, which can support cancer growth. The myofibroblastic phenotype of CAFs in cancer micro-environment is TGF- $\beta$-SMAD signaling dependent. It is also reported that phosphatidylserine mediated fusion of exosomes and endothelial cell facilitate the expression of EGFR on cancer associated endothelial cells. Also, TAMs secret the VEGF into cancer microenvironment to regulate angiogenesis. Exosomes play a pivotal role in maintains a balance between pro- and anti-angiogenic factors the cancer microenvironment [102].

\section{Exosomes remodeling host extracellular matrix}

Exosome binding can alter cancer microenvironment by modulating the host extracellular matrix (ECM). Their abundant enzymes affect the host matrix by degrading proteins [117]. Exosomes from a highly metastatic rat pancreatic cancer line (ASML cells) have recovered high levels of uPAR, MMP2, MMP3, MMP9, MMP14, and ADAM17 proteinases. In vitro experiments demonstrate that ASML ${ }^{\text {wt }}$ (ASML wild type cells without any molecular modifications) exosomal proteases are functional degradation for the ECM of stromal lines and endothelial cells, and for several proteins as collagen, fibronectin and laminins [32, 33]. Furthermore, exosomal proteases may contribute to matrix protein maturation such as the ADAMTS family for collagen maturation [118]. Also, they are possibly involved in generating fragments of matrix proteins to exert distinct functions such as laminin fragment promoting cell motility and

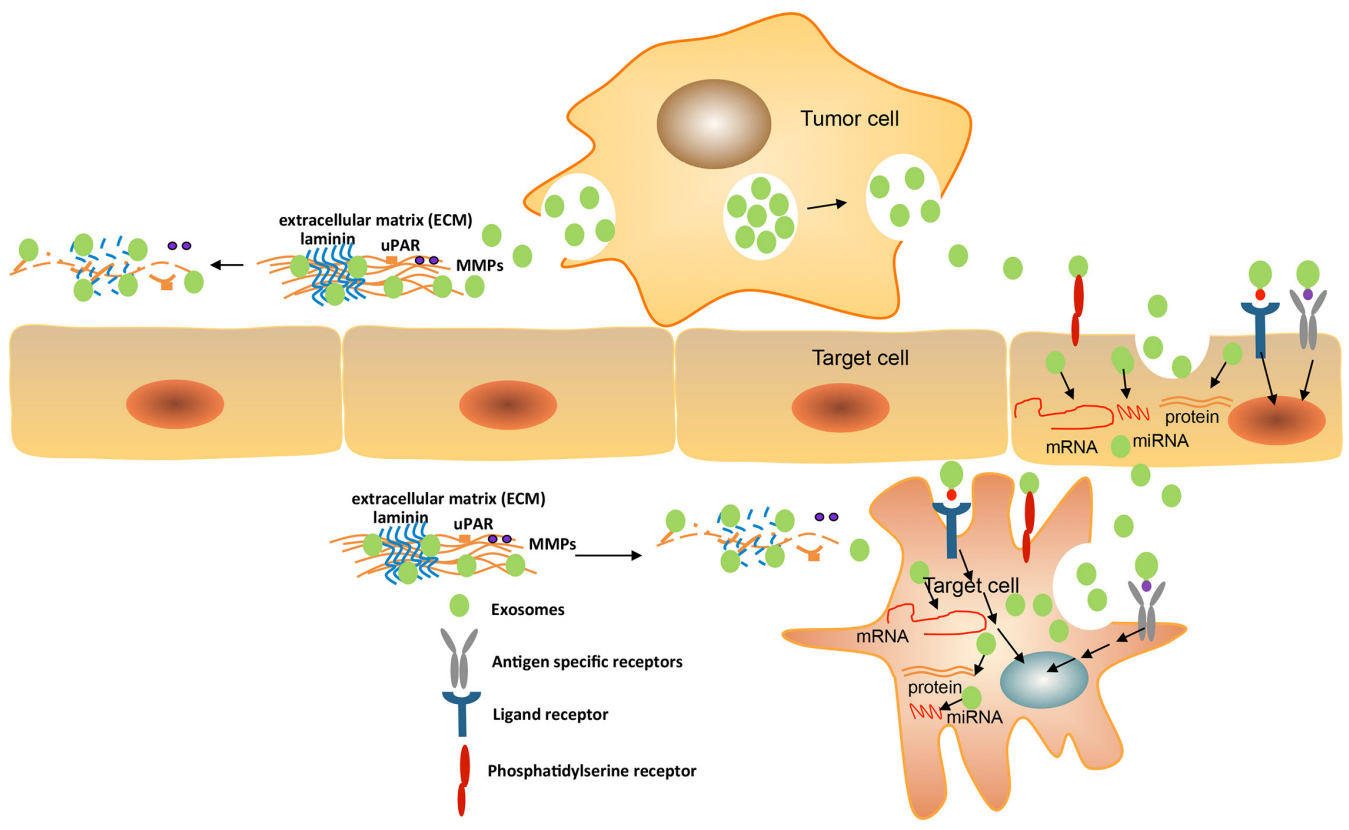

Figure 3: Exosomes mediate target cell reprogramming and alter cancer microenvironment. Exosomes can uptake into the target cells (endothelia cell or cancer stroma cell) by the membrane fusion mechanisms, the interaction of phosphatidyl serine and its receptor, the binding of ligands and receptors, and the transfer of both the specific molecules and antigens. Exosomes can directly bind cell surface receptors by interaction with exosomal ligands that may activate the cascade of signaling transduction, thus initiates the expression of specific genes by transcription factors regulation, oncogenes activity, and the immune regulation. Exosomes containing various molecules including proteins, antigen and cytokines can be delivered to recipient cells during internalization that allow regulating target cells. Exosomal mRNAs and miRNAs are functional and can express into new proteins or regulate specific gene expression in recipient cells. The exosomal components may mediate target cell reprogramming. In addition, exosomes enriched complex of tetraspanin/ proteinase including MMPs, uPAR, TACE, and ADAM17 with full activity regulate cell motility and invasiveness via extracellular matrix degradation. The matrix proteins as laminins, collagens may be degraded by proteinase to create a path for cell migration or invasion. 
small HA fragments promoting an inflammatory milieu $[119,120]$. Taken together, exosomal proteases are critical elements for cross talk between tumors and the host matrix by creating space for cell motility and cancer metastasis (Figure 3).

Cancer cell line AS, expressing Tspan8-derived exosomes, promotes endothelial cell proliferation and cable formation. Co-culture of endothelial cell and AS-Tspan8 exosomes induces migration-related expression of molecules as CCR1, CCL20, CXCL5, and MIF [102]. MIF may stabilize HIF1 $\alpha$ to promote the expression of angiogenic growth factor [121]. Similar angiogenic effects were observed with exosomes derived from different tumor lines expressing Tspan 8 and integrin $\alpha 4$. This indicated that the exosomal Tspan 8 integrin $\alpha 4$ complex was a decisive factor in exosomes-induced angiogenesis without the defined angiogenic factors. Angiogenesis induction is independent of the release of angiogenic factors but essentially depends on Tspan8-containing exosomes. As consistent components of exosomes, tetraspanins present a clue for the exosomal angiogenic function.

Animal experiments demonstrated that the highly metastatic line ASML has a slow local and metastatic growth in lymph nodes and lungs. A CD44 variant isoform selective knockdown of this line (ASML-CD44 $\mathrm{v}^{\mathrm{kd}}$ ) loses the capacity of metastasis formation [122]. The exosomes secreted by the knockdown line do not exert the fully metastatic phenotype while their co-cultures facilitate knockdown cells settling in lymph nodes and lungs. ASML cell-derived exosomes include enriched tetraspanins, integrin $\alpha 6 \beta 4$, EpCAM, claudin-7, c-Met, uPAR, MMPs, and other molecules. Results indicated the association of integrin $\alpha 6 \beta 4$, EpCAM and claudin-7 with Tspan8 in ASML exosomes. ASML exosomes upregulate several adhesion molecules including integrin $\alpha 4$, CD44v6, chemokines, growth factors, angiogenic factors and proteases as well as preferentially targeted lungs and lymph node stromal cells $[123,124]$. The double knockdown of ASML (ASML ${ }^{\mathrm{dk}}$-Tsp8-CD151) by Tspan8 and CD151 lacks the capacity of metastasis [125]. ASML exosomes can recover the metastatic capacity of ASML ${ }^{\mathrm{dk}}$ Tsp8-CD151 and ASML-CD44vkd cell [33]. These studies demonstrated how ASML-derived exosomes support premetastatic niche formation. Tetraspanins are involved in the preparation of premetastatic niche formation.

\section{CONCLUSION}

Exosomes are small vesicles with large functions and play a pivotal role in inter as well as intracellular communications. Though the complex role of exosomes in cancer biology is slowly being established, knowledge on their mode of interaction with the cancer microenvironment is still limited. It is well known that exosomes recruit diverse bioactive molecules including proteins,
mRNA and miRNA of donor cells. However, little is known as to how exosomes influence distant cells or alter the cancer micro-environment. The enrichment of tetraspanins in exosomes may provide a clue to uncover the mechanism of their selective uptake by target cells. The central feature of tetraspanins is a molecular facilitator that associates with other tetraspanins, integrins and additional transmembrane proteins in TEM. Also, it facilitates the recruitment of signal molecules. TEM assembly in exosomes surface and internalize tetraspanins is through the process of endocytosis. Exosomal tetraspanins direct the selection of target cells and the preparation of pre-metastatic niche formation. The complex content of exosomes exerts the reprogramming of target cells by translating new protein and regulating new gene expression.

The nano-scale particle of exosomes is extensively beneficial to apply to the process of diagnosis and therapeutics. Tumor-derived exosomes in all body fluids may provide an alternative for a reliable diagnostic tool. Cancer-specific exosomal protein and miRNA have received much attention for being possible diagnosis markers. The potential of delivering therapeutic content and biological membranes without invoking an immune response drives exosomes to be the new cargo for therapeutics. As the most important intercellular communicators, exosomes modulate, instruct, and reprogramme their surroundings as well as distant organs. The tailored exosomes may offer a potent therapeutic delivery system. In the last several years, research on exosomes have attracted an ever-increasing and overwhelming interest. The knowledge of exosomes will open new doors in the field of cancer biology including diagnosis and therapy.

\section{ACKNOWLEDGMENTS}

I mostly thank to Prof. Margot Zoller for her suggestions and help for this article preparation. I am grateful to the people of my laboratory. The authors would like to thank and express the apology for many outstanding contributions to the field, which could not be cited in case of limited space.

\section{CONFLICTS OF INTEREST}

The authors declare that they have no conflicts of interest.

\section{GRANT SUPPORT}

The work in the authors laboratory of this article was supported by the National Key Scientific Projects of China (2013CB967200) and NSFC General Project (81470354) to RX, the Natural Science Foundation of Tianjin, China (16JCYBJC43200) to SY. 


\section{REFERENCES}

1. Chaffer CL, Weinberg RA. A perspective on cancer cell metastasis. Science. 2011; 331:1559-1564.

2. Sund M, Kalluri R. Tumor stroma derived biomarkers in cancer. Cancer Metastasis Rev. 2009; 28:177-183.

3. Marvel D, Gabrilovich DI. Myeloid-derived suppressor cells in the tumor microenvironment: expect the unexpected. J Clin Invest. 2015; 125:3356-3364.

4. Chang CH, Qiu J, O’Sullivan D, Buck MD, Noguchi T, Curtis JD, Chen Q, Gindin M, Gubin MM, van der Windt GJ, Tonc E, Schreiber RD, Pearce EJ, Pearce EL. Metabolic competition in the tumor microenvironment is a driver of cancer progression. Cell. 2015; 162:1229-1241.

5. Folkman J. Endogenous angiogenesis inhibitors. APMIS. 2004; 112:496-507.

6. Legg JA, Herbert JM, Clissold P, Bicknell R. Slits and Roundabouts in cancer, tumor angiogenesis and endothelial cell migration. Angiogenesis. 2008; 11:13-21.

7. Ribatti D, Nico B, Crivellato E, Vacca A. Macrophages and tumor angiogenesis. Leukemia. 2007; 21:2085-2089.

8. Hughes CC. Endothelial-stromal interactions in angiogenesis. Curr Opin Hematol. 2008; 15:204-209.

9. Duda DG, Jain RK. Premetastatic lung "niche": is vascular endothelial growth factor receptor 1 activation required? Cancer Res. 2010; 70:5670-5673.

10. Kaplan RN, Riba RD, Zacharoulis S, Bramley AH, Vincent L, Costa C, MacDonald DD, Jin DK, Shido K, Kerns SA, Zhu Z, Hicklin D, Wu Y, et al. VEGFR1-positive haematopoietic bone marrow progenitors initiate the premetastatic niche. Nature. 2005; 438:820-827.

11. Costa-Silva B, Aiello NM, Ocean AJ, Singh S, Zhang H, Thakur BK, Becker A, Hoshino A, Mark MT, Molina H, Xiang J, Zhang T, Theilen TM, et al. Pancreatic cancer exosomes initiate pre-metastatic niche formation in the liver. Nat Cell Biol. 2015; 17:816-826.

12. Hemler ME. Tetraspanin functions and associated microdomains. Nat Rev Mol Cell Biol. 2005; 6:801-811.

13. Zhang L, Zhang S, Yao J, Lowery FJ, Zhang Q, Huang WC, Li P, Li M, Wang X, Zhang C, Wang H, Ellis K, Cheerathodi $\mathrm{M}$, et al. Microenvironment-induced PTEN loss by exosomal microRNA primes brain metastasis outgrowth. Nature. 2015; 527:100-104.

14. Hemler ME. Tetraspanin proteins promote multiple cancer stages. Nat Rev Cancer. 2014; 14:49-60.

15. Schneider A, Simons M. Catching filopodia: exosomes surf on fast highways to enter cells. J Cell Biol. 2016; 213:143-145.

16. Desrochers LM, Antonyak MA, Cerione RA. Extracellular vesicles: satellites of information transfer in cancer and stem cell biology. Dev Cell. 2016; 37:301-309.
17. Szempruch AJ, Sykes SE, Kieft R, Dennison L, Becker AC, Gartrell A, Martin WJ, Nakayasu ES, Almeida IC, Hajduk SL, Harrington JM. Extracellular vesicles from trypanosoma brucei mediate virulence factor transfer and cause host anemia. Cell. 2016; 164:246-257.

18. Saleem SN, Abdel-Mageed AB. Tumor-derived exosomes in oncogenic reprogramming and cancer progression. Cell Mol Life Sci. 2015; 72:1-10.

19. Zomer A, Maynard C, Verweij FJ, Kamermans A, Schafer R, Beerling E, Schiffelers RM, de Wit E, Berenguer J, Ellenbroek SI, Wurdinger T, Pegtel DM, van Rheenen J. In Vivo imaging reveals extracellular vesicle-mediated phenocopying of metastatic behavior. Cell. 2015; 161:1046-1057.

20. Colombo M, Moita C, van Niel G, Kowal J, Vigneron J, Benaroch P, Manel N, Moita LF, Thery C, Raposo G. Analysis of ESCRT functions in exosome biogenesis, composition and secretion highlights the heterogeneity of extracellular vesicles. J Cell Sci. 2013; 126:5553-5565.

21. Baietti MF, Zhang Z, Mortier E, Melchior A, Degeest G, Geeraerts A, Ivarsson Y, Depoortere F, Coomans C, Vermeiren E, Zimmermann P, David G. Syndecan-synteninALIX regulates the biogenesis of exosomes. Nat Cell Biol. 2012; 14:677-685.

22. Colombo M, Raposo G, Thery C. Biogenesis, secretion, and intercellular interactions of exosomes and other extracellular vesicles. Ann Rev Cell Dev Biol. 2014; 30:255-289.

23. Minciacchi VR, Freeman MR, Di Vizio D. Extracellular vesicles in cancer: exosomes, microvesicles and the emerging role of large oncosomes. Semin Cell Dev Biol. 2015; 40:41-51.

24. Zhang J, Li S, Li L, Li M, Guo C, Yao J, Mi S. Exosome and exosomal microRNA: trafficking, sorting, and function. Genomics Proteomics Bioinformatics. 2015; 13:17-24.

25. Munich S, Sobo-Vujanovic A, Buchser WJ, Beer-Stolz D, Vujanovic NL. Dendritic cell exosomes directly kill tumor cells and activate natural killer cells via TNF superfamily ligands. Oncoimmunology. 2012; 1:1074-1083.

26. Mulcahy LA, Pink RC, Carter DR. Routes and mechanisms of extracellular vesicle uptake. J Extracell Vesicles. 2014; 3.

27. Tian T, Zhu YL, Hu FH, Wang YY, Huang NP, Xiao ZD. Dynamics of exosome internalization and trafficking. J Cell Physiol. 2013; 228:1487-1495.

28. Quesenberry PJ, Aliotta J, Deregibus MC, Camussi G. Role of extracellular RNA-carrying vesicles in cell differentiation and reprogramming. Stem Cell Res Ther. 2015; 6:153.

29. Roberts CT Jr, Kurre P. Vesicle trafficking and RNA transfer add complexity and connectivity to cell-cell communication. Cancer Res. 2013; 73:3200-3205.

30. Aliotta JM, Pereira M, Johnson KW, de Paz N, Dooner MS, Puente N, Ayala C, Brilliant K, Berz D, Lee D, Ramratnam B, McMillan PN, Hixson DC, et al. Microvesicle entry into 
marrow cells mediates tissue-specific changes in mRNA by direct delivery of mRNA and induction of transcription. Expt Hematol. 2010; 38:233-245.

31. Rana S, Yue S, Stadel D, Zoller M. Toward tailored exosomes: the exosomal tetraspanin web contributes to target cell selection. Int J Biochem Cell Biol. 2012; 44:1574-1584.

32. Yang C, Robbins PD. The roles of tumor-derived exosomes in cancer pathogenesis. Clin Dev Immunol. 2011; 2011:842849.

33. $\mathrm{Mu} \mathrm{W}$, Rana S, Zoller M. Host matrix modulation by tumor exosomes promotes motility and invasiveness. Neoplasia. 2013; 15:875-887.

34. Yue S, Mu W, Erb U, Zoller M. The tetraspanins CD151 and Tspan8 are essential exosome components for the crosstalk between cancer initiating cells and their surrounding. Oncotarget. 2015; 6:2366-2384. doi: 10.18632/ oncotarget.2958.

35. Tkach M, Thery C. Communication by extracellular vesicles: where we are and where we need to go. Cell. 2016; 164:1226-1232.

36. Liu Y, Gu Y, Cao X. The exosomes in tumor immunity. Oncoimmunology. 2015; 4:e1027472.

37. Zech D, Rana S, Buchler MW, Zoller M. Tumor-exosomes and leukocyte activation: an ambivalent crosstalk. Cell Commun Signal. 2012; 10:37.

38. Peng P, Yan Y, Keng S. Exosomes in the ascites of ovarian cancer patients: origin and effects on anti-tumor immunity. Oncol Rep. 2011; 25:749-762.

39. Fan GC. Hypoxic exosomes promote angiogenesis. Blood. 2014; 124:3669-3670.

40. Ekstrom EJ, Bergenfelz C, von Bulow V, Serifler F, Carlemalm E, Jonsson G, Andersson T, Leandersson K. WNT5A induces release of exosomes containing proangiogenic and immunosuppressive factors from malignant melanoma cells. Mol Cancer. 2014; 13:88.

41. Hu Y, Yan C, Mu L, Huang K, Li X, Tao D, Wu Y, Qin J. Fibroblast-derived exosomes contribute to chemoresistance through priming cancer stem cells in colorectal cancer. PLoS One. 2015; 10:e0125625.

42. Gruenberg J, Stenmark H. The biogenesis of multivesicular endosomes. Nat Rev Mol Cell Biol. 2004; 5:317-323.

43. Johnstone RM, Adam M, Hammond JR, Orr L, Turbide C. Vesicle formation during reticulocyte maturation. Association of plasma membrane activities with released vesicles (exosomes). J Biol Chem. 1987; 262:9412-9420.

44. Rothman JE. Mechanisms of intracellular protein transport. Nature. 1994; 372:55-63.

45. Denzer K, Kleijmeer MJ, Heijnen HF, Stoorvogel W, Geuze HJ. Exosome: from internal vesicle of the multivesicular body to intercellular signaling device. J Cell Sci. 2000; 113 Pt 19:3365-3374.

46. Urbanelli L, Magini A, Buratta S, Brozzi A, Sagini K, Polchi A, Tancini B, Emiliani C. Signaling pathways in exosomes biogenesis, secretion and fate. Genes. 2013; 4:152-170.

47. Lakkaraju A, Rodriguez-Boulan E. Itinerant exosomes: emerging roles in cell and tissue polarity. Trends Cell Biol. 2008; 18:199-209.

48. Kowal J, Tkach M, Thery C. Biogenesis and secretion of exosomes. Curr Opin Cell Biol. 2014; 29:116-125.

49. Johnstone RM. Exosomes biological significance: a concise review. Blood Cell Mol Dis. 2006; 36:315-321.

50. Zhang HG, Grizzle WE. Exosomes: a novel pathway of local and distant intercellular communication that facilitates the growth and metastasis of neoplastic lesions. Am J Pathol. 2014; 184:28-41.

51. Ding Q, Chen J, Wei X, Sun W, Mai J, Yang Y, Xu Y. RAFTsomes containing epitope-MHC-II complexes mediated CD4 $+\mathrm{T}$ cell activation and antigen-specific immune responses. Pharm Res. 2013; 30:60-69.

52. EL Andaloussi S, Mager I, Breakefield XO, Wood MJ. Extracellular vesicles: biology and emerging therapeutic opportunities. Nat Rev Drug Discov. 2013; 12:347-357.

53. Luarte A, Batiz LF, Wyneken U, Lafourcade C. Potential therapies by stem cell-derived exosomes in cns diseases: focusing on the neurogenic niche. Stem Cell Int. 2016; 2016:5736059.

54. Schorey JS, Bhatnagar S. Exosome function: from tumor immunology to pathogen biology. Traffic. 2008; 9:871-881.

55. Valadi H, Ekstrom K, Bossios A, Sjostrand M, Lee JJ, Lotvall JO. Exosome-mediated transfer of mRNAs and microRNAs is a novel mechanism of genetic exchange between cells. Nat Cell Biol. 2007; 9:654-659.

56. Fabian MR, Sonenberg N, Filipowicz W. Regulation of mRNA translation and stability by microRNAs. Ann Rev Biochem. 2010; 79:351-379.

57. Goldie BJ, Dun MD, Lin M, Smith ND, Verrills NM, Dayas CV, Cairns MJ. Activity-associated miRNA are packaged in Map1b-enriched exosomes released from depolarized neurons. Nucleic Acids Res. 2014; 42:9195-9208.

58. Gajos-Michniewicz A, Duechler M, Czyz M. MiRNA in melanoma-derived exosomes. Cancer Lett. 2014; 347:29-37.

59. Fang Y, Wu N, Gan X, Yan W, Morrell JC, Gould SJ. Higher-order oligomerization targets plasma membrane proteins and HIV gag to exosomes. PLoS Biol. 2007; 5:e158.

60. Andre F, Schartz NE, Chaput N, Flament C, Raposo G, Amigorena S, Angevin E, Zitvogel L. Tumor-derived exosomes: a new source of tumor rejection antigens. Vaccine. 2002; 20:A28-31.

61. Zoller M. Gastrointestinal tumors: metastasis and tetraspanins. Z Gastroenterol. 2006; 44:573-586.

62. Andreu Z, Yanez-Mo M. Tetraspanins in extracellular vesicle formation and function. Front Immunol. 2014; $5: 442$. 
63. Taylor DD, Gercel-Taylor C. MicroRNA signatures of tumor-derived exosomes as diagnostic biomarkers of ovarian cancer. Gynecol Oncol. 2008; 110:13-21.

64. Madhavan B, Yue S, Galli U, Rana S, Gross W, Muller M, Giese NA, Kalthoff H, Becker T, Buchler MW, Zoller M. Combined evaluation of a panel of protein and miRNA serum-exosome biomarkers for pancreatic cancer diagnosis increases sensitivity and specificity. Int J Cancer. 2015; 136:2616-2627.

65. Mitchell PS, Parkin RK, Kroh EM, Fritz BR, Wyman SK, Pogosova-Agadjanyan EL, Peterson A, Noteboom J, O'Briant KC, Allen A, Lin DW, Urban N, Drescher CW, et al. Circulating microRNAs as stable blood-based markers for cancer detection. Proc Natl Acad Sci U S A. 2008; 105:10513-10518.

66. Tanaka Y, Kamohara H, Kinoshita K, Kurashige J, Ishimoto T, Iwatsuki M, Watanabe M, Baba H. Clinical impact of serum exosomal microRNA-21 as a clinical biomarker in human esophageal squamous cell carcinoma. Cancer. 2013; 119:1159-1167.

67. Rabinowits G, Gercel-Taylor C, Day JM, Taylor DD, Kloecker GH. Exosomal microRNA: a diagnostic marker for lung cancer. Clin Lung Cancer. 2009; 10:42-46.

68. Wright MD, Geary SM, Fitter S, Moseley GW, Lau LM, Sheng KC, Apostolopoulos V, Stanley EG, Jackson DE, Ashman LK. Characterization of mice lacking the tetraspanin superfamily member CD151. Mol Cell Biol. 2004; 24:5978-5988.

69. Zhang Z, Wang F, Li Q, Zhang H, Cui Y, Ma C, Zhu J, $\mathrm{Gu}$ X, Sun Z. CD151 knockdown inhibits osteosarcoma metastasis through the GSK-3beta/beta-catenin/MMP9 pathway. Oncol Rep. 2016; 35:1764-1770.

70. Li Q, Yang XH, Xu F, Sharma C, Wang HX, Knoblich K, Rabinovitz I, Granter SR, Hemler ME. Tetraspanin CD151 plays a key role in skin squamous cell carcinoma. Oncogene. 2013; 32:1772-1783.

71. Bobrie A, Colombo M, Raposo G, Thery C. Exosome secretion: molecular mechanisms and roles in immune responses. Traffic. 2011; 12:1659-1668.

72. Kanetaka K, Sakamoto M, Yamamoto Y, Takamura M, Kanematsu T, Hirohashi S. Possible involvement of tetraspanin CO-029 in hematogenous intrahepatic metastasis of liver cancer cells. J Gastroenterol Hepatol. 2003; 18:1309-1314.

73. Garcia-Espana A, Chung PJ, Sarkar IN, Stiner E, Sun TT, Desalle R. Appearance of new tetraspanin genes during vertebrate evolution. Genomics. 2008; 91:326-334.

74. Hemler ME. Tetraspanin proteins mediate cellular penetration, invasion, and fusion events and define a novel type of membrane microdomain. Ann Rev Cell Dev Biol. 2003; 19:397-422.

75. Park CS, Kim TK, Kim HG, Kim YJ, Jeoung MH, Lee WR, Go NK, Heo K, Lee S. Therapeutic targeting of tetraspanin8 in epithelial ovarian cancer invasion and metastasis. Oncogene. 2016; 35:4540-4548.

76. Tang M, Yin G, Wang F, Liu H, Zhou S, Ni J, Chen C, Zhou Y, Zhao Y. Downregulation of CD9 promotes pancreatic cancer growth and metastasis through upregulation of epidermal growth factor on the cell surface. Oncol Rep. 2015; 34:350-358.

77. Feng J, Huang C, Wren JD, Wang DW, Yan J, Zhang J, Sun Y, Han X, Zhang XA. Tetraspanin CD82: a suppressor of solid tumors and a modulator of membrane heterogeneity. Cancer Metastasis Rev. 2015; 34:619-633.

78. Berditchevski F, Odintsova E. Tetraspanins as regulators of protein trafficking. Traffic. 2007; 8:89-96.

79. Zoller M. Tetraspanins: push and pull in suppressing and promoting metastasis. Nat Rev Cancer. 2009; 9:40-55.

80. Perez-Hernandez D, Gutierrez-Vazquez C, Jorge I, LopezMartin S, Ursa A, Sanchez-Madrid F, Vazquez J, Yanez-Mo $\mathrm{M}$. The intracellular interactome of tetraspanin-enriched microdomains reveals their function as sorting machineries toward exosomes. J Biol Chem. 2013; 288:11649-11661.

81. Le Naour F, Andre M, Boucheix C, Rubinstein E. Membrane microdomains and proteomics: lessons from tetraspanin microdomains and comparison with lipid rafts. Proteomics. 2006; 6:6447-6454.

82. Zhang XA, Bontrager AL, Hemler ME. Transmembrane-4 superfamily proteins associate with activated protein kinase $\mathrm{C}$ (PKC) and link PKC to specific beta(1) integrins. J Biol Chem. 2001; 276:25005-25013.

83. Berditchevski F. Complexes of tetraspanins with integrins: more than meets the eye. J Cell Sci. 2001; 114:4143-4151.

84. Winterwood NE, Varzavand A, Meland MN, Ashman LK, Stipp CS. A critical role for tetraspanin CD151 in alpha3beta1 and alpha6beta4 integrin-dependent tumor cell functions on laminin-5. Mol Biol Cell. 2006; 17:2707-2721.

85. Hasegawa M, Furuya M, Kasuya Y, Nishiyama M, Sugiura T, Nikaido T, Momota Y, Ichinose M, Kimura S. CD151 dynamics in carcinoma-stroma interaction: integrin expression, adhesion strength and proteolytic activity. Lab Invest. 2007; 87:882-892.

86. Takeda Y, Kazarov AR, Butterfield CE, Hopkins BD, Benjamin LE, Kaipainen A, Hemler ME. Deletion of tetraspanin Cd151 results in decreased pathologic angiogenesis in vivo and in vitro. Blood. 2007; 109:1524-1532.

87. Rana S, Claas C, Kretz CC, Nazarenko I, Zoeller M. Activation-induced internalization differs for the tetraspanins CD9 and Tspan8: impact on tumor cell motility. Int J Biochem Cell Biol. 2011; 43:106-119.

88. Khatun Z, Bhat A, Sharma S, Sharma A. Elucidating diversity of exosomes: biophysical and molecular characterization methods. Nanomedicine. 2016; 11:2359-2377.

89. Akiel MA, Santhekadur PK, Mendoza RG, Siddiq A, Fisher PB, Sarkar D. Tetraspanin 8 mediates AEG-1-induced 
invasion and metastasis in hepatocellular carcinoma cells. FEBS Lett. 2016; 590:2700-2708.

90. Gesierich S, Berezovskiy I, Ryschich E, Zoller M. Systemic induction of the angiogenesis switch by the tetraspanin D6.1A/CO-029. Cancer Res. 2006; 66:7083-7094.

91. Iwasaki T, Takeda Y, Maruyama K, Yokosaki Y, Tsujino K, Tetsumoto S, Kuhara H, Nakanishi K, Otani Y, Jin Y, Kohmo S, Hirata H, Takahashi R, et al. Deletion of tetraspanin CD9 diminishes lymphangiogenesis in vivo and in vitro. J Biol Chem. 2013; 288:2118-2131.

92. Bass R, Werner F, Odintsova E, Sugiura T, Berditchevski F, Ellis V. Regulation of urokinase receptor proteolytic function by the tetraspanin CD82. J Biol Chem. 2005; 280:14811-14818.

93. Pols MS, Klumperman J. Trafficking and function of the tetraspanin CD63. Exp Cell Res. 2009; 315:1584-1592.

94. Huotari J, Helenius A. Endosome maturation. EMBO J. $2011 ; 30: 3481-3500$.

95. Hicke L, Dunn R. Regulation of membrane protein transport by ubiquitin and ubiquitin-binding proteins. Ann Rev Cell Dev Biol. 2003; 19:141-172.

96. Hurley JH. ESCRT complexes and the biogenesis of multivesicular bodies. Curr Opin Cell Biol. 2008; 20:4-11.

97. Wollert T, Hurley JH. Molecular mechanism of multivesicular body biogenesis by ESCRT complexes. Nature. 2010; 464:864-869.

98. Gruenberg J, van der Goot FG. Mechanisms of pathogen entry through the endosomal compartments. Nat Rev Mol Cell Biol. 2006; 7:495-504.

99. Holowka D, Sheets ED, Baird B. Interactions between $\mathrm{Fc}($ epsilon)RI and lipid raft components are regulated by the actin cytoskeleton. J Cell Sci. 2000; 113:1009-1019.

100. Dutta D, Williamson CD, Cole NB, Donaldson JG. Pitstop 2 is a potent inhibitor of clathrin-independent endocytosis. PLoS One. 2012; 7:e45799.

101. Abache T, Le Naour F, Planchon S, Harper F, Boucheix C, Rubinstein $\mathrm{E}$. The transferrin receptor and the tetraspanin web molecules CD9, CD81, and CD9P-1 are differentially sorted into exosomes after TPA treatment of K562 cells. J Cell Biochem. 2007; 102:650-664.

102. Nazarenko I, Rana S, Baumann A, McAlear J, Hellwig A, Trendelenburg M, Lochnit G, Preissner KT, Zoller M. Cell surface tetraspanin Tspan8 contributes to molecular pathways of exosome-induced endothelial cell activation. Cancer Res. 2010; 70:1668-1678.

103. Mazurov D, Barbashova L, Filatov A. Tetraspanin protein CD9 interacts with metalloprotease CD10 and enhances its release via exosomes. FEBS J. 2013; 280:1200-1213.

104. Simpson F, Hussain NK, Qualmann B, Kelly RB, Kay BK, McPherson PS, Schmid SL. SH3-domain-containing proteins function at distinct steps in clathrin-coated vesicle formation. Nat Cell Biol. 1999; 1:119-124.

105. Koh TW, Korolchuk VI, Wairkar YP, Jiao W, Evergren E, Pan H, Zhou Y, Venken KJ, Shupliakov O, Robinson IM,
O'Kane CJ, Bellen HJ. Eps15 and Dap160 control synaptic vesicle membrane retrieval and synapse development. J Cell Biol. 2007; 178:309-322.

106. Rana S, Zoller M. Exosome target cell selection and the importance of exosomal tetraspanins: a hypothesis. Biochem Soc Trans. 2011; 39:559-562.

107. Smalheiser NR. Exosomal transfer of proteins and RNAs at synapses in the nervous system. Biol Direct. 2007; 2:35.

108. Camussi G, Deregibus MC, Bruno S, Grange C, Fonsato V, Tetta C. Exosome/microvesicle-mediated epigenetic reprogramming of cells. Am J Cancer Res. 2011; 1:98-110.

109. Syn N, Wang L, Sethi G, Thiery JP, Goh BC. Exosomemediated metastasis: from epithelial-mesenchymal transition to escape from immunosurveillance. Trends Pharmacol Sci. 2016; 37:606-617.

110. Kahlert C, Kalluri R. Exosomes in tumor microenvironment influence cancer progression and metastasis. J Mol Med. 2013; 91:431-437.

111. Hoshino A, Costa-Silva B, Shen TL, Rodrigues G, Hashimoto A, Tesic Mark M, Molina H, Kohsaka S, Di Giannatale A, Ceder S, Singh S, Williams C, Soplop N, et al. Tumor exosome integrins determine organotropic metastasis. Nature. 2015; 527:329-335.

112. Yuan A, Farber EL, Rapoport AL, Tejada D, Deniskin R, Akhmedov NB, Farber DB. Transfer of microRNAs by embryonic stem cell microvesicles. PLoS One. 2009; 4:e4722.

113. Donnarumma E, Fiore D, Nappa M, Roscigno G, Adamo A, Iaboni M, Russo V, Affinito A, Puoti I, Quintavalle C, Rienzo A, Piscuoglio S, Thomas R, Condorelli G. Cancerassociated fibroblasts release exosomal microRNAs that dictate an aggressive phenotype in breast cancer. Oncotarget. 2017; 8:19592-19608. doi: 10.18632/ oncotarget.14752.

114. Zhou S, Abdouh M, Arena V, Arena M, Arena GO. Reprogramming malignant cancer cells toward a benign phenotype following exposure to human embryonic stem cell microenvironment. PLoS One. 2017; 12:e0169899.

115. Cai Z, Yang F, Yu L, Yu Z, Jiang L, Wang Q, Yang Y, Wang L, Cao X, Wang J. Activated T cell exosomes promote tumor invasion via Fas signaling pathway. J Immunol. 2012; 188:5954-5961.

116. Kim JW, Wieckowski E, Taylor DD, Reichert TE, Watkins $\mathrm{S}$, Whiteside TL. Fas ligand-positive membranous vesicles isolated from sera of patients with oral cancer induce apoptosis of activated $\mathrm{T}$ lymphocytes. Clin Cancer Res. 2005; 11:1010-1020.

117. Mathivanan S, Ji H, Simpson RJ. Exosomes: extra-cellular organelles important in intercellular communication. J Proteomics. 2010; 73:1907-1920.

118. Apte SS. A disintegrin-like and metalloprotease (reprolysintype) with thrombospondin type 1 motif (ADAMTS) superfamily: functions and mechanisms. J Biol Chem. 2009; 284:31493-31497. 
119. Malinda KM, Wysocki AB, Koblinski JE, Kleinman HK, Ponce ML. Angiogenic laminin-derived peptides stimulate wound healing. Int J Biochem Cell Biol. 2008; 40:2771-2780.

120. Cantor JO, Nadkarni PP. Hyaluronan: the Jekyll and Hyde molecule. Inflamm Allergy Drug Targets. 2006; 5:257-260.

121. Rendon BE, Willer SS, Zundel W, Mitchell RA. Mechanisms of macrophage migration inhibitory factor (MIF)-dependent tumor microenvironmental adaptation. Exp Mol Pathol. 2009; 86:180-185.

122. Jung T, Gross W, Zoller M. CD44v6 coordinates tumor matrix-triggered motility and apoptosis resistance. J Biol Chem. 2011; 286:15862-15874.
123. Zoller M. CD44: can a cancer-initiating cell profit from an abundantly expressed molecule? Nat Rev Cancer. 2011; 11:254-267.

124. Jung T, Castellana D, Klingbeil P, Cuesta Hernandez I, Vitacolonna M, Orlicky DJ, Roffler SR, Brodt P, Zoller M. CD44v6 dependence of premetastatic niche preparation by exosomes. Neoplasia. 2009; 11:1093-1105.

125. Yue S, Mu W, Zoller M. Tspan8 and CD151 promote metastasis by distinct mechanisms. Eur J Cancer. 2013; 49:2934-2948. 